\title{
CURRENTS IN, FORCES ON AND DEFORMATIONS/DISPLACEMENTS OF THE LHC BEAM SCREEN EXPECTED DURING A MAGNET QUENCH
}

\author{
C. Rathjen, F. Caspers, P. Pugnat, S. Russenschuck, A. Siemko, CERN, Geneva, Switzerland
}

\section{Abstract}

Due to the field asymmetry of the LHC dipoles, the magnetic field integral calculated from the centre of the aperture to the outside of the cold mass does not vanish. During a magnet quench this asymmetry generates an electromotive force and thus a current with a resultant lateral force on the beam screen. This induced force was observed indirectly when measuring the deformation of the beam screen cross-section during a quench using a precision displacement transducer, which will be described. The transducer (based on optical gratings) was developed specially to study the beam screen deformation in cryogenic environments and high magnetic fields. The results of the measurements are compared to calculations and to direct measurements of the induced voltage along the current path. An estimation of the forces exerted on the cold bore by the beam screen and of the actual current induced in the beam screen will be given.

\section{INTRODUCTION}

Fig. 1 shows a section through one beam pipe (cold bore tube) of a LHC dipole magnet. The main purpose of the beam screen is to reduce the beam induced heat input into the cold mass of the super conducting magnet and to insure vacuum stability. To reduce the impedance of the LHC machine, a thin copper layer is required on the inside of the stainless steel beam screen. One drawback of the desired low impedance is that it facilitates the induction of eddy currents (mainly in the copper layer because of its very low resistance at $20 \mathrm{~K}$ ). As a consequence, the decaying magnetic field generates Lorentz forces which expand the beam screen horizontally (see Fig. 2).

According to measurements and calculations [1], the Lorentz forces are approximately proportional to $B \mathrm{~d} B / \mathrm{d} t$ (flux density $B$, Fig.2). For a quench at nominal field of $8.3 \mathrm{~T}$ maximal Lorentz forces in the order of $4 \mathrm{~N} / \mathrm{mm}^{3}$ are generated $190 \mathrm{~ms}$ after the quench started. The maximum sum of all Lorentz forces in one half of the beam screen is approximately $10 \mathrm{kN} / \mathrm{m}$. At $210 \mathrm{~ms}$ the sum of all eddy currents reaches its maximum of about $1.8 \mathrm{kA}$.

As no transducers for high magnetic fields and cryogenic environments (also small enough for the beam screen) were available, CERN's vacuum group developed displacement transducers (Fig. 3) to measure quench induced deformations in a precise and reliable manner [2].

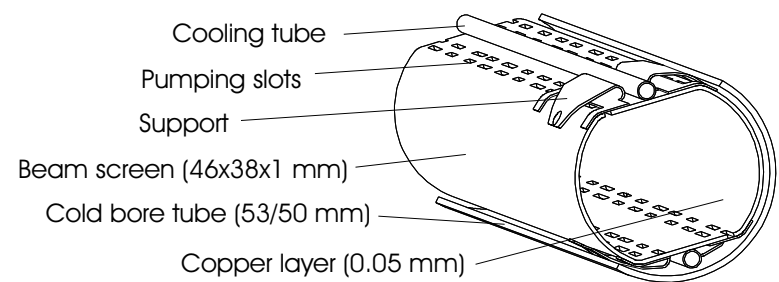

Figure 1: Test beam screen inside cold bore tube.

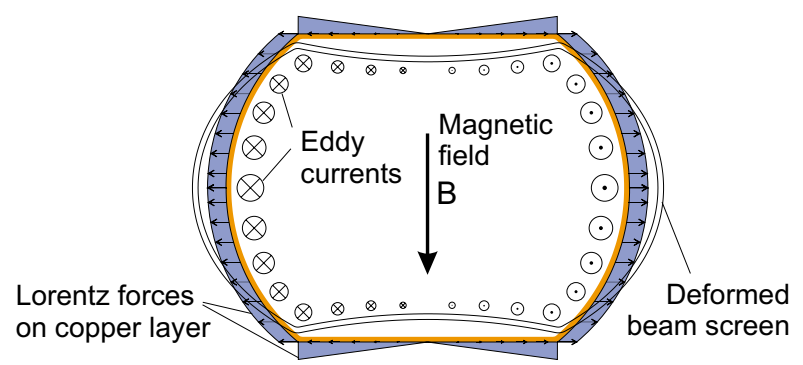

Figure 2: Induced eddy currents and resulting deformation of a beam screen during a LHC dipole quench.
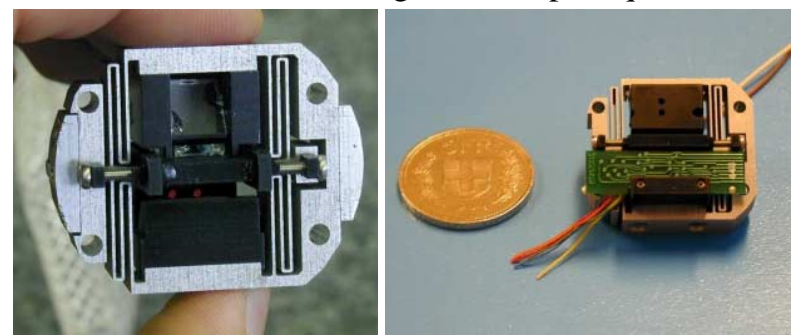

Figure 3: Optical displacement transducers with optical fibres (left) and with attached electronics.

\section{MEASUREMENTS}

Quenches under different initial conditions were performed in a $15 \mathrm{~m}$ long LHC dipole magnet prototype [3] (beam screen temperatures: 6 30 K ; magnetic field $<9$ T). Fig. 3 shows the two transducers used for the measurements. One transducer had its electronics (IRLED and photodiodes) inside the magnetic field. Since the transducer electronics could not be pre-tested under quench conditions, a second transducer was equipped with optical fibres to measure fully independent of cryogenic temperatures and high magnetic fields; the visible diode laser and 4 photodiodes were installed inside the cryostats insulation vacuum close to room temperature but outside the magnetic field. Finally both transducers performed well under quench conditions.

\subsection{Instrumentation}

The optical transducer is based on the method of counting fringes on an optical grating (that is used as a measurement scale). Fig. 4 shows the essential parts of the transducer version with optical fibres. The measurement grating (fixed on one side of the beam screen) is subjected to the displacement to be measured. The grating consists of $10 \mu \mathrm{m}$ broad fringes of chrome, having a pitch of $20 \mu \mathrm{m}$, on a glass substrate. A detector, fixed on the other side of the beam screen, counts the fringes passing by. Principal components of the detector are a collimated light source, a reference grating of the same pitch and a receiver with photodiodes. The reference grating consist of four $90^{\circ}$ phase shifted gratings which 
enable a more sophisticated fringe counting with interpolation that can also distinguish between forward and backward movement. The measurement grating has to be guided parallel to the reference grating with high precision. The guidance is realised by a parallelogram of four folded leaf springs, which make the transducer hysteresis free and avoid jamming at cold.

The advantage of the transducer is the use of periodic signals. Even if the signals are distorted the possible error stays within the signal period of $20 \mu \mathrm{m}$ as long as fringe counting is possible. Since the deformation to be measured is coded in the phase of the signals rather than in the amplitude, a synchronous amplitude or offset change does not affect the phase measurement if 4 phase shifted signals are processed. However, this type of transducer is very sensitive to misalignment. If the measurement and the reference grating are not properly aligned parallel, the signal modulation drops practically to zero. As a consequence the risk of a total system failure is high, but, if signals are received, a high precision of the measurement is guaranteed.

These advantages allow for transducers that do not have to be calibrated at cold if the thermal contraction of the grating material is sufficiently well known. Having a well adjusted set-up a precision better than 1 micron can be achieved due to the high quality of the gratings in use.

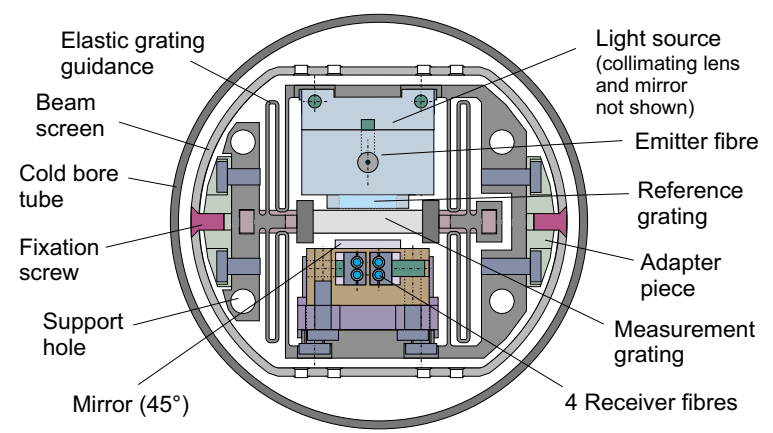

Fig. 4: Optical transducer mounted inside beam screen.

\subsection{Beam screen deformation during quench}

Fig. 5 shows a sine-cosine signal pair generated (by subtracting the $180^{\circ}$ phase shifted signals) of the transducer with electronics inside the magnetic field during a quench at nominal field. This signal pair was demodulated and unwrapped using common phase measuring techniques. Offset removal and amplitude equalisation were done by software keeping errors less than one eighth of the grating pitch without cumbersome fine-tuning of the transducer. Fig. 6 shows the resulting deformations of the beam screen for the same quench.

The maximal error of these measurements was determined including all possible sources to be less than $3 \mu \mathrm{m}$ for the electronic version and less than $6 \mu \mathrm{m}$ for the version with optical fibres (due to smaller sensing apertures of the read out fibres and not corrected errors mentioned in Fig. 7). The high quality of the signals in Fig. 5 insured that no fringe counting errors occurred. Even one signal was sufficient to determine the highest deformation (period counts, comp. Fig. 5).

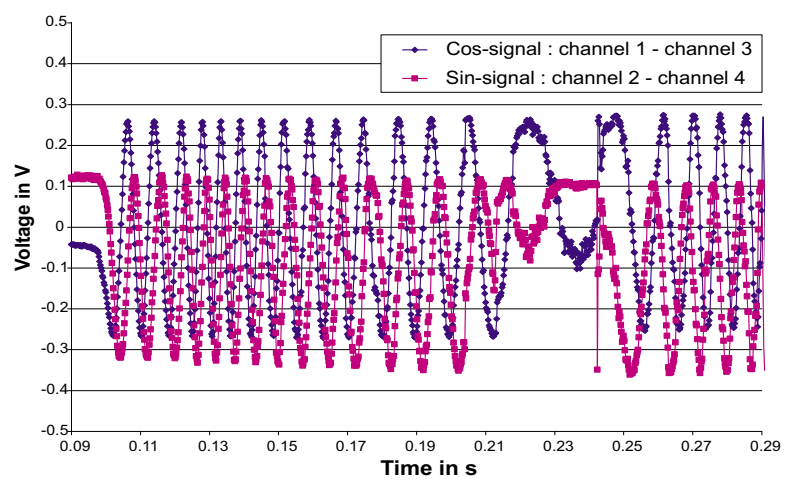

Figure 5 : Signal pair from transducer with electronics

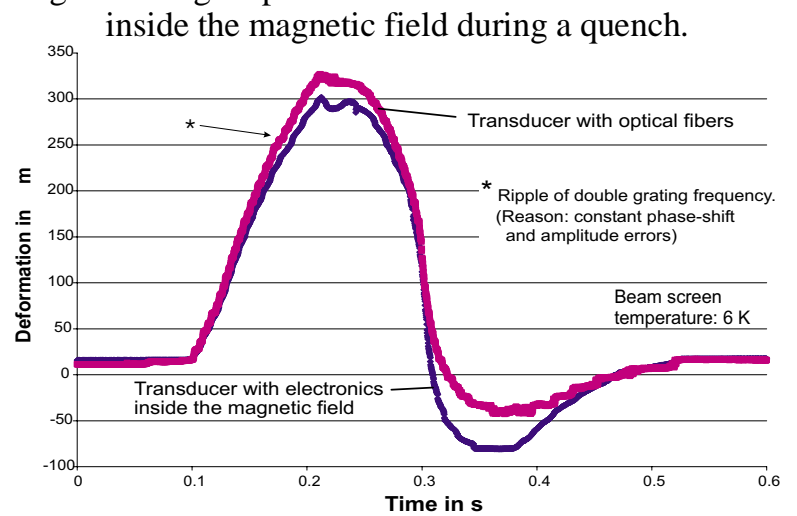

Figure 6: Horizontal deformation of beam screen during a quench measured at two different locations.

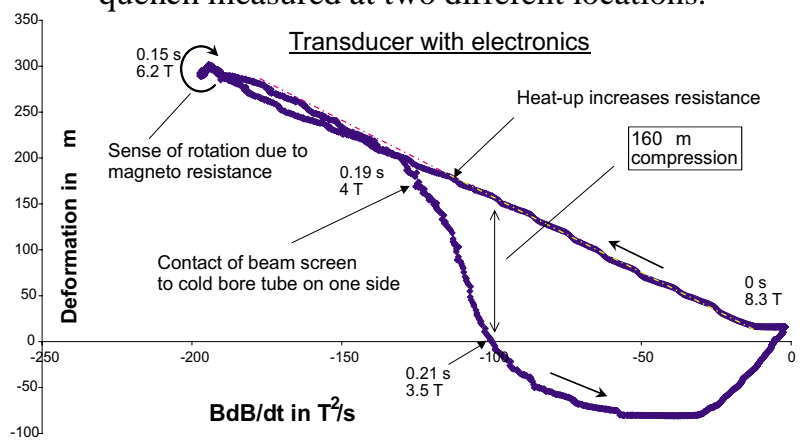

Figure 7: Beam screen deformation versus $B \mathrm{~d} B / \mathrm{d} t$.

\subsection{Discussion of the measurements}

For the first time reliable deformation measurements with a known precision could be performed on a beam screen at working conditions. Measurements with strain gauges [4] and resistors performed in the past were in agreement (rising slopes and maximum deformations were consistent). A new observation was a compression of the beam screen at the end of the quench (see Fig. 6). Since the model of Fig. 2 could not describe compression, an explanation had to be found.

To investigate the phenomena, Fig. 7 shows the deformation plotted versus $B \mathrm{~d} B / \mathrm{d} t$ which is approximately proportional to the Lorentz forces. At the beginning of the quench the beam screen deforms as expected from past measurements. Comparison with simulations could identify slight effects thanks to the high precision of the transducer (Fig. 7: change of slope due to heat up of the beam screen, sense of rotation due to 
decreasing magneto resistance). About $190 \mathrm{~ms}$ after quench begin, the beam screen leaves the expected (straight) path and is compressed. This compression persists until the magnetic forces have vanished.

After the quench test, the beam screen was dismounted to generate a comparable compression in the lab. Neither vibration nor bending tests gave compressions in the order of $-100 \mu \mathrm{m}$. Only a compression of the beam screen against a cold bore tube could generate this signal. The high reliability of the transducer left as only possible explanation for the undershoot a contact of the beam screen with the cold bore tube at the end of the quench.

\section{MODEL OF BEAM SCREEN CONTACT}

A slightly unbalanced magnetic field in the magnet yoke was found as an explanation. Fig. 8 shows a section of a LHC dipole cold mass. Indicated in the figure is the loop formed by the beam screen and the external cylinder of the magnets cold mass. The integral of the magnetic flux density across the loop is not equal to zero. Thus a change of the magnetic field induces a current $I$ in the loop (superimposed to the eddy currents in Fig. 2). This current in combination with the magnetic field in the aperture generates an inward directed force on the beam screen that is responsible for the lateral contact and the subsequent compression of the beam screen during a quench.

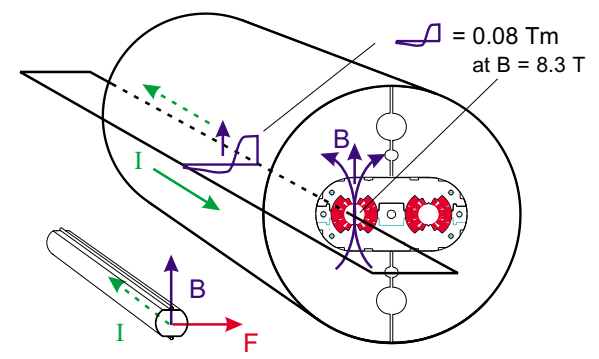

Figure 8: Model to describe the contact of the beam screen to the cold bore tube.

\section{CURRENTS AND FORCES}

According to calculations, the integral of the flux density over a $14.3 \mathrm{~m}$ long loop, as indicated in Fig. 8, is equal to $1.145 \mathrm{Tm}^{2}$ at the nominal field of $8.3 \mathrm{~T}$. Furthermore, calculations demonstrate a proportionality of the integral to the magnetic field in the aperture. Using this linearity the induced voltage can be expressed as $U_{\text {ind }}=\mathrm{d} B / \mathrm{d} t 0.138 \mathrm{~m}^{2}$. Taking the maximal field decay in the aperture of $34 \mathrm{~T} / \mathrm{s}$ the induced voltage is about $4.7 \mathrm{~V}$.

Assuming a loop resistance of $15 \mathrm{~m} \Omega$ results in a maximal current along the beam screen of about $313 \mathrm{~A}$. This value corresponds quite well to measurements performed with a real loop when ramping a magnet (up to $5 \mathrm{~A} / \mathrm{s}$ and $1 \mathrm{kA}$ ). About $330 \mathrm{~A}$ were estimated for the current [5]. Both currents result in a contact force of about $1650 \mathrm{~N} / \mathrm{m}$ at the maximal $\mathrm{d} B / \mathrm{d} t$ at $5 \mathrm{~T}$.

Considering that the beam screen is an elastic structure, the width of the loop in Fig. 7 can be taken to determine the contact force. $210 \mathrm{~ms}$ after quench begin, at the moment of the highest field decay, $160 \mu \mathrm{m}$ compression can be seen in Fig. 7. Referring to the compression tests performed after the quench tests, this corresponds to a contact force of $1300 \mathrm{~N} / \mathrm{m}$. Considering the acting magnetic field of $3.5 \mathrm{~T}$ results in a current of about $365 \mathrm{~A}$. This calculation assumes a contact force proportional to $B \mathrm{~d} B / \mathrm{d} t$. Therefore this contact force has to be scaled up to the maximal $B \mathrm{~d} B / \mathrm{d} t$, which results in $2000 \mathrm{~N} / \mathrm{m}$. However, this high value is not likely to be present during a quench since the contact happens after $B \mathrm{~d} B / \mathrm{d} t$ has reached its maximum (see Fig. 7). At the moment there is no explanation for this time delay.

A second approach is to take the load due to eddy currents at maximum deformation $(7500 \mathrm{~N} / \mathrm{m}$ in each curved side of the beam screen at $300 \mu \mathrm{m}$ ) to deduce the contact force. At the moment when the current I compensates the eddy currents on one side of the beam screen the deformation should be zero. In Fig. 7 this is the case $210 \mathrm{~ms}$ after quench begin. At this time Lorentz forces have halved to $3750 \mathrm{~N} / \mathrm{m}$. This values represents rather an upper limit for the contact force since the transducer's fixation screws were elevated above the beam screen contour by $0.6 \mathrm{~mm}$, thus causing signal amplification due to force concentration. Tests are foreseen to quantify this signal amplification.

\section{CONCLUSIONS}

During a nominal quench the LHC dipole beam screen expands horizontally by $300 \mu \mathrm{m}$. Shortly after maximal expansion, eddy currents induced in the beam screen reach their maximum of about $1.8 \mathrm{kA}$. Superimposed to these eddy currents, an additional current along the beam screen, estimated to be $350 \mathrm{~A}$, is induced in an external loop. At the end of the quench, Lorentz forces generated by this current in the order of $1500 \mathrm{~N} / \mathrm{m}$ move and compress the beam screen against the cold bore tube. Since the dynamics of this effect are not fully understood yet, investigations are going on. Direct current measurements in the interconnects of the LHC test cell ("String 2") will also investigate the propagation of this additional current to neighbouring components and magnets.

\section{REFERENCES}

[1] C. Rathjen, "A model to calculate LHC beam screen deformation in a dipole field during quench", Vac. Tech. Note 00-21, CERN LHC/VAC, Dec. 2000.

[2] C. Rathjen, "Optical displacement transducer for applications at cryogenic temperatures and high magnetic fields", Vac. Tech. Note 00-22, CERN LHC/VAC, Dec. 2000.

[3] J. Martinez-Darve et al., "Measurement of the mechanical behaviour of the LHC beam screen during a quench", PAC, Session WPAH035, June 2001.

[4] C. Rathjen, "First quench tests of a prototype LHC racetrack type beam screen in a short dipole magnet model", Tech. Note CERN EST-ESI/99-13.

[5] P. Pugnat et al., "Induced voltages measured across wire loops in a full scale LHC-dipole", LHC-MTAIN-2000-142, CERN, Dec. 2000. 